\title{
Expression of ADAM29 and FAM135B in the pathological evolution from normal esophageal epithelium to esophageal cancer: Their differences and clinical significance
}

\author{
TINGTING WANG ${ }^{1}$, XIAOYAN LV ${ }^{1}$, SHEN JIANG ${ }^{2}$, SHAORONG HAN $^{1}$ and YANMING WANG ${ }^{1}$ \\ ${ }^{1}$ Department of Radiation Oncology, PLA 960th Hospital, Jinan, Shandong 250031; \\ ${ }^{2}$ Department of Emergency Internal Medicine, Shanghai East Hospital, \\ Tongji University School of Medicine, Shanghai 201203, P.R. China
}

Received January 26, 2019; Accepted November 17, 2019

DOI: $10.3892 / \mathrm{ol} .2020 .11272$

\begin{abstract}
A Disintegrin And Metalloprotease Domain 29 (ADAM29) and Family with sequence similarity 135 member B (FAM135B) genes have been reported to be associated with a carcinogenic risk of esophageal squamous cell carcinoma (ESCC). However, to the best of our knowledge, the expression of ADAM29 and FAM135B in the pathological evolution from normal esophageal epithelial cells to ESCC has not yet been investigated. The present study aimed to investigate the expression of ADAM29 and FAM135B in normal esophageal mucosal epithelium, low-grade and high-grade esophageal intraepithelial neoplasia, and ESCC. Furthermore, the present study aimed to investigate the role of ADAM29 and FAM135B in the development of esophageal lesions. Immunohistochemistry was performed in order to detect the expression levels of ADAM29 and FAM135B proteins in normal esophageal mucosa samples (40 cases), low-grade intraepithelial neoplasia samples (20 cases), high-grade intraepithelial neoplasia samples (20 cases) and ESCC samples (40 cases). The results of the present study demonstrated that the positive rates of ADAM29 and FAM135B proteins increased gradually from normal esophageal mucosal epithelium and esophageal intraepithelial neoplasia, to ESCC $(\mathrm{P}<0.05)$. Furthermore, the expression levels of ADAM29 and FAM135B proteins in ESCC were not associated with age and the tumor size $(\mathrm{P}>0.05)$; however, the protein levels were associated with the pathological stage, clinical stage and lymph node metastasis of ESCC $(\mathrm{P}<0.05)$. In addition, there was a significant association between the expression levels of ADAM29 protein and FAM135B protein $\left(\chi^{2}=60.071 ; \mathrm{P}<0.001\right)$. The results of the present study demonstrated that the expression levels
\end{abstract}

Correspondence to: Dr Yanming Wang, Department of Radiation Oncology, PLA 960th Hospital, 25 Shifan Road, Jinan, Shandong 250031, P.R. China

E-mail: ymwang64@163.com

Key words: esophageal, squamous cell carcinoma, intraepithelial neoplasis, A Disintegrin And Metalloprotease Domain 29, Family with sequence similarity 135 -member B of ADAM29 and FAM135B were associated with the tumor behavior characteristics and the progression of esophageal cancer, the expression of which could be used for the diagnosis of early esophageal cancer, and provide the basis for guiding individualized treatment.

\section{Introduction}

Esophageal squamous cell carcinoma (ESCC) is a common malignancy of the digestive system. In 2018, the incidence of esophageal cancer ranked seventh among malignancies, and there was a total of 572,034 new cases (1). The epidemiology of ESCC is not only associated with country, dietary habit, ethnicity and sex, but its incidence is significantly different within different regions $(2,3)$. According to the global cancer data released by the American Cancer Society in 2012, East Asia had the highest mortality rate of esophageal cancer, and its incidence and mortality rates ranked fifth (age-standardized incidence rate, $7.74 \%$ ) and fourth (age-standardized mortality rate, $9.29 \%$ ), respectively among malignancies in China (4). Esophageal cancer is predominantly divided into squamous cell carcinoma and adenocarcinoma, the latter of which is more common in western countries. However, squamous cell carcinoma dominates in China, accounting for $\sim 90 \%$ of all reported cases of esophageal cancer (5), and Northern Jiangsu possesses one of the highest incidences of esophageal cancer in China and in 2006, the incidences rate of esophageal cancer was 86.45/100,000 in Chuzhou (Jiangsu, China) $(2,6)$. According to the cancer data report registered in Huai'an City, esophageal cancer accounts for $32.02 \%$ of all malignancies, and the incidence and mortality of ESCC in the Huai'an area have been among the highest of all malignancies in the last 10 years (7). Despite advancements in the treatment of esophageal cancer, the prognosis of patients with esophageal cancer remains poor, whereby the 5-year survival rate is $<20 \%$ (8). However, in early stage esophageal cancer, the 5-year survival rate can be as high as $80-90 \%$ (9), and $40 \%$ of esophageal cancer is prone to recurrence (10). Due to the concealment and non-specificity of the early symptoms of esophageal cancer, the majority of patients are diagnosed and treated in the middle or advanced 
stages of disease (11). ESCC and adenocarcinoma exhibit different pathogeneses, biological tumor characteristics and prognoses (12). Therefore, adopting the foreign standard may not be suitable for patients with esophageal cancer in China, as it would do more harm than good. Further studies are required in order to develop the diagnosis and treatment plans according to the characteristics of ESCC in China, in order to improve diagnosis of early stage esophageal cancer (13).

The continuous development of molecular biology and an improved understanding of its underlying mechanisms has subsequently allowed for advancements to the development of tumor markers for the assessment of tumor treatment efficacy, recurrence monitoring and prognosis. Song et al (14) demonstrated that eight genes were associated with ESCC, including six well-known oncology-associated genes: p53, RB1, CDKN2, PIK3CA, NOTCH1 and NF2L2. Furthermore, to the best of our knowledge, A Disintegrin And Metalloprotease Domain 29 (ADAM29) and Family With Sequence Similarity 135-member B (FAM135B) were demonstrated to be associated with ESCC for the first time.

A member of the ADAMs family, ADAM29 is a type-I transmembrane protein located in human chromosome $4 q 34.1$ (15), which plays an important role in regulating cell-to-cell or cell-matrix interactions (16). ADAMs are widely involved in a number of physiological processes, including sperm-egg fusion, nervous system development and inflammatory response (17). Furthermore, ADAMs play an important role in the invasion and metastasis of malignancies, Victor et al (18) demonstrated that ADAM9 participates in the vascular metastasis process of pancreatic ductal adenocarcinoma. Besides, ADAM12 promotes the metastasis of esophageal cancer by influencing the interaction between tumor cells and the extracellular matrix (19) and ADAM17 participates in Notch and Wnt signaling pathways to promotes metastasis in gastric cancer (20). ADAMs are also known as metalloproteinase depolymerization (21). Zhang et al (22) indicated that ADAM29 was associated with breast cancer subtypes, and it was also detected in non-small cell lung cancer (23). In a study on gastric cancer, it was revealed that ADAM29 influenced gastric carcinoma proliferation, migration, invasion and motility via upregulation of the MKN45-C1 and -C2 clones, and the MKN45-SC control (24). Brim et al (25) determined that ADAM29 is associated with colorectal tumors, and it has also been revealed that the ADAM29 mutation affects the adhesion of melanoma cells to specific extracellular matrix proteins and promotes tumor invasion (26). Conversely, the FAM135B gene is located in human chromosome 8q24.23 (27). A number of studies have demonstrated that the FAM135B gene is associated with the occurrence and development of tumor, and can promote the proliferation, migration and invasion of tumor cells $(11,14,28)$.

To the best of our knowledge, the expression of ADAM29 and FAM135B proteins in the precancerous stage of esophageal cancer has not yet been reported. Immunohistochemistry (IHC) was performed in the present study in order to investigate the differences and clinical significance of the expression of ADAM29 and FAM135B in the pathological evolution from normal esophageal epithelium to esophageal cancer.

\section{Materials and methods}

Human esophagus specimens. A total of 120 esophageal paraffin mass specimens were collected from 80 patients with ESCC at the Department of Pathology of the 82nd Hospital of the People's Liberation Army (Huai'an, China) between January 2013 to January 2015. There were 40 cases of esophageal intraepithelial neoplasia following biopsy (20 cases of high-grade esophageal intraepithelial neoplasia and 20 cases of low-grade esophageal intraepithelial neoplasia) and 40 cases of post-surgical ESCC, of which 21 cases were $>60$ years old and 19 cases $\leq 60$ years old. A total of 40 cases of normal mucosal tissue of the corresponding esophageal carcinoma $(\geq 5 \mathrm{~cm}$ from the tumor border) were treated as the control group for ESCC. The pathological classification were as follows: A total of 19 cases in the G1 stage (29-30), 19 cases in the G2 stage and two cases in the G3 stage. The clinical stages were as follows: A total of eight cases in stage I (29-30), 22 cases in stage II: 10 cases in stage III. Lymph node metastasis was reported in 10 cases and absent in 30 cases. The tumor size were as follows: $>5 \mathrm{~cm}$ in two patients and $\leq 5 \mathrm{~cm}$ in 38 patients. There was an equal sex distribution among the pathological grades of esophageal cancer. The American Joint Committee on Cancer (AJCC) 2010 (31) was adopted in order to develop the standard manual for tumor staging. The inclusion criteria were as follows: i) Patient had no prior history of cancer; ii) samples submitted for examination and materials must be sufficient; iii) radiotherapy and/or chemotherapy had not been performed; iv) inflammatory diseases were not combined; and v) specimens were confirmed repeatedly by pathology (confirmed by two or more specialist pathologists). The present study was approved by the People's Liberation Army 82nd Hospital Ethics Committee. All patients, or their families, provided written informed consent prior to the study start.

Reagents. Rabbit anti-human ADAM29 polyclonal antibody (1:50; cat. no. ab198875) was purchased from Abcam. Rabbit anti-human FAM135B polyclonal antibody (1:50; cat. no. 51059) was purchased from Sigma-Aldrich (Merck KGaA). The secondary antibody kit [maxvison ${ }^{\mathrm{TM}} 2$ HRP (Mouse/Rabbit) IHC kit; cat. no. KIT5920; Ready-to-use antibody] and DAB (cat. no. DAB-2031/2032) dye were purchased from Fuzhou Maixin Biotech Co, Ltd. The secondary antibody kit was used to amplify the signal of the primary antibody, and the dye was used to locate, characterize and quantify intracellular antigens.

Immunohistochemistry. All postoperative or biopsy specimens were fixed for $24 \mathrm{~h}$ with $10 \%$ phosphate buffer formalin fixation solution and embedded in paraffin at $20^{\circ} \mathrm{C}$. Paraffin-embedded tissues were cut into sections ( $3 \mu \mathrm{m}$-thick) for use in routine pathological examination and immunohistochemical examination. All immunohistochemical methods were performed using the Elivision two-step method (32). Briefly, the following procedures were performed: Specimens were baked at $70^{\circ} \mathrm{C}$ for $1 \mathrm{~h}$, before being dewaxed in xylene for $20 \mathrm{~min}$ and rehydrated with graded concentrations of alcohol for $17 \mathrm{~min}$. Slices were placed in solution of citrate $(\mathrm{pH} \mathrm{6.0)}$ and heated in a pressure cooker (1.03 kpa) for $3 \mathrm{~min}, 3 \% \mathrm{H}_{2} \mathrm{O}_{2}$ was added and incubated at room temperature for $15 \mathrm{~min}$ to block endogenous peroxidase. Sections were then incubated for $60 \mathrm{~min}$ at 
$37^{\circ} \mathrm{C}$ with primary antibodies (Rabbit anti-human ADAM29 polyclonal antibody; 1:50; cat. no. ab198875; Abcam) and phosphate buffer was used as a negative control. Second antibody [maxvisonTM2 HRP (Mouse/Rabbit) IHC kit; Ready-to-use antibody; cat. no. KIT5920; Sigma-Aldrich; Merck KGaA] was added and incubated at room temperature for $20 \mathrm{~min}$. Slices were stained using DAB at room temperature for $5 \mathrm{~min}$, redyed with hematoxylin at room temperature for $20 \mathrm{sec}$ and differentiated with $1 \%$ hydrochloric acid alcohol for $1 \mathrm{sec}$ at room temperature.

Result judgement. The degree of section coloring was observed using a high-power optical light microscope (magnification, $\mathrm{x} 400$ ). A double-blind method was implemented in order to observe the esophageal tissue staining. A total of 10 high-power visual fields on each slice were randomly-selected and counted, and at least 100 cells were observed in each field. The positive expression of ADAM29 protein was predominantly concentrated in the cell membrane or cytoplasm, while the positive expression of FAM135B protein was predominantly concentrated in the nucleus. Esophageal tissue appeared brown as the positive expression marker of ADAM29 and FAM135B proteins in the glass slice. The comprehensive score was obtained, according to the staining intensity of the esophageal tissue and the proportion of positive cells. The staining intensity score were as follows: Zero indicated no coloring, 1 indicated light yellow, 2 indicated yellow-brown and 3 indicated brown. Stained tissues were counted in five randomly-selected fields using a high-power optical light microscope (magnification, x400), and at least 100 cells were observed in each field. The percentage score of positive cell slices were as follows: $0,<5 \%$ positive cells; $1,5-25 \%, 2,26-50 \% ; 3,51-75 \%$; and $4,>75 \%$. The product of two scores were as follows: $0-1$ indicated a negative score (-), 2-5 indicated a positive score $(+), 6-8$ indicated a positive score $(++) /(2+)$ and $\geq 9$ indicated a strong positive score $(+++) /(3+)$; low-positive expression group $(\geq 2$ and $<9)$ and high-positive expression group ( $\geq 9)$.

Statistical analysis. All data were analyzed using SPSS software (version 22.0; IBM Corp.). Categorical variables were presented as frequencies and percentages. Categorical variables were compared with $\chi^{2}$ tests or Fisher exact tests (Tables I and II). Relevance between categorical variables were performed using $\chi^{2}$ tests (Table III). $\mathrm{P}<0.05$ was considered to indicate a statistically significant difference.

\section{Results}

Expression of ADAM29 protein in different stages of esophageal lesions. Overall, ADAM29 protein was negatively expressed in normal esophageal epithelial cells; however, weak positive expression was occasionally demonstrated in the cytoplasm of basal cells. Furthermore, the staining was relatively shallow in normal esophageal epithelial cells, but the majority of ESCC cells appeared brown. ADAM29 expression in esophageal lesions was concentrated in the cytoplasm of squamous cell carcinoma (Fig. 1). The expression of ADAM29 protein gradually enhanced from normal esophageal mucosal epithelium and intraepithelial esophageal neoplasia, to ESCC.
Furthermore, ADAM29 staining gradually deepened and the staining area gradually increased in these groups (Fig. 1). The positive expression rate of ADAM29 was $17.5 \%$ in normal esophageal tissue, $60.0 \%$ in low-grade intraepithelial neoplasia tissue, $90.0 \%$ in high-grade intraepithelial neoplasia tissue and $100.0 \%$ in ESCC tissue (Fig. 2). The differences in distribution among the four groups were statistically significant (Table I; $\mathrm{P}<0.05)$.

Expression of FAM135B protein in different stages of esophageal lesions. FAM135B protein was expressed in certain normal epithelial basal cells of the esophagus, and appeared light yellow. The majority of tumor cells in the esophageal cancer tissues appeared brown, and the positive expression was concentrated in the nucleus of squamous cell carcinoma (Fig. 1). The expression of FAM135B protein gradually increased from the normal esophageal mucosal epithelium and intraepithelial esophageal neoplasia, to ESCC. Furthermore, FAM135B staining gradually deepened and the staining area gradually increased in these groups (Fig. 1). The positive expression rate of FAM135B was $25 \%$ in normal esophageal tissue, $55 \%$ in low-grade intraepithelial neoplasia tissue, $90 \%$ in high-grade intraepithelial neoplasia tissue and 100\% in ESCC (Fig. 2). The differences in distribution among the four groups were statistically significant (Table I; $\mathrm{P}<0.05$ ).

Association between the expression of ADAM29 and FAM135B proteins and the severity of esophageal epithelial cell lesions. The positive rates of ADAM29 and FAM135B proteins gradually increased from normal esophageal mucosal epithelium and intraepithelial esophageal neoplasia, to ESCC (Table I; $\mathrm{P}<0.05$ ).

Association between the expression of ADAM29 and FAM135B proteins and the clinic-pathological features of $E S C C$. Patients with esophageal cancer were divided into the high expression group $(\geq 9)$ and the low expression group $(\geq 2$ and $<9$ ), according to the product of two scores of percentage (positive cell slices and staining intensity) as presented in Table II, the cutoff value was a score of 9.

Association between the expression of ADAM29 protein and the clinicopathological features of ESCC. As presented in Table II, increased ADAM29 expression was significantly associated with the lymph node metastasis features of ESCC ( $\mathrm{P}=0.003)$. Furthermore, high ADAM29 expression was significantly associated with the pathological stage $(\mathrm{P}=0.024)$ and clinical stage $(\mathrm{P}=0.016)$. The case of ADAM29 expression in each pathological stage, two cases $(10.5 \%)$ were observed in G1, nine cases $(47.4 \%)$ in $\mathrm{G} 2$ and one case $(50 \%)$ in G3. High ADAM29 expression occurred in zero cases $(0 \%)$ in clinical stage I, six cases $(27.3 \%)$ in clinical stage II: six cases $(60 \%)$ in clinical stage III. High expression of ADAM29 was not associated with patient age $(\mathrm{P}=0.629)$ and tumor size $(\mathrm{P}=0.515)$.

Association between the expression of FAM135B protein and the clinicopathological features of ESCC. Increased FAM135B expression was significantly associated with the lymph node metastasis features of ESCC $(\mathrm{P}=0.002)$. 
Table I. Expression of ADAM29 and FAM135B in different esophageal lesions.

\begin{tabular}{lccc}
\hline Tissue type & Cases, $\mathrm{n}$ & Positive rate of ADAM29, $\mathrm{n}(\%)$ & Positive rate of FAM135B, $\mathrm{n}(\%)$ \\
\hline Normal squamous epithelium & 40 & $7(17.5)$ & $10(25.0)$ \\
Low-grade intraepithelial neoplasia & 20 & $12(60.0)$ & $11(55.0)$ \\
High-grade intraepithelial neoplasia & 20 & $18(90.0)$ & $18(90.0)$ \\
Esophageal cancer & 40 & $40(100.0)$ & $40(100.0)$ \\
$\chi^{2}$ & & 66.179 & 56.647 \\
P-value & & $<0.001$ & $<0.001$ \\
\hline
\end{tabular}

ADAM29, A Disintegrin And Metalloprotease Domain 29; FAM135B, Family with sequence similarity 135-member B.

Table II. Baseline characteristics of study populations.

\begin{tabular}{|c|c|c|c|c|c|c|c|}
\hline Characteristic & Cases, n & $\begin{array}{c}\text { ADAM29 (score, } 3+), \\
\text { n }(\%)\end{array}$ & $\mathrm{D}^{2}$ & P-value & $\begin{array}{c}\text { FAM135B (score, } 3+) \\
\mathrm{n}(\%)\end{array}$ & $\mathrm{A}^{2}$ & P-value \\
\hline Age & & & & & & & 0.115 \\
\hline$\leq 60$ years & 21 & $7(33.3)$ & 0.234 & 0.629 & $8(38.1)$ & 2.489 & \\
\hline$>60$ years & 19 & $5(26.3)$ & & & $3(15.8)$ & & \\
\hline Pathological grade & & & & & & & 0.006 \\
\hline G1 & 19 & $2(10.5)$ & 6.903 & 0.024 & $1(5.3)$ & 9.636 & \\
\hline $\mathrm{G} 2$ & 19 & $9(47.4)$ & & & $9(47.4)$ & & \\
\hline G3 & 2 & $1(50.0)$ & & & $1(50)$ & & \\
\hline Clinical stage & & & & & & & 0.015 \\
\hline I & 8 & $0(0.0)$ & 7.414 & 0.016 & $0(0.0)$ & 7.836 & \\
\hline II & 22 & $6(27.3)$ & & & $5(22.7)$ & & \\
\hline III & 10 & $6(60.0)$ & & & $6(60.0)$ & & \\
\hline Lymph node metastasis & & & & & & & 0.002 \\
\hline No & 30 & $5(16.7)$ & 10.159 & 0.003 & $4(13.8)$ & 12.079 & \\
\hline Yes & 10 & $7(70.0)$ & & & $7(63.6)$ & & \\
\hline Tumor size & & & & & & & 0.515 \\
\hline$\leq 5 \mathrm{~cm}$ & 38 & $11(22.4)$ & 0.401 & 0.515 & $11(22.4)$ & 0.401 & \\
\hline$>5 \mathrm{~cm}$ & 2 & $1(50.0)$ & & & $1(50.0)$ & & \\
\hline
\end{tabular}

ADAM29, A Disintegrin And Metalloprotease Domain 29; FAM135B, Family with sequence similarity 135-member B. (3+) indicates a strong positive score and $\mathrm{n}$ represents the number of positive expressions in group.

Furthermore, high FAM135B expression was significantly associated with the pathological stage $(\mathrm{P}=0.006)$ and clinical stage $(\mathrm{P}=0.015)$. The case of FAM135B expression in each pathological stage, one case $(5.30 \%)$ was observed in $\mathrm{G} 1$, nine cases (47.4\%) in G2 and one case $(50 \%)$ in G3. High FAM135B expression occurred in zero cases $(0 \%)$ in clinical stage I, five cases $(22.7 \%)$ in clinical stage II: six cases $(60 \%)$ in clinical stage III. High expression of FAM135B was not associated with patient age $(\mathrm{P}=0.115)$ or tumor size $(\mathrm{P}=0.515)$.

Association between ADAM29 and FAM135B protein expressions. The association between ADAM29 and FAM135B protein expressions was assessed using $\chi^{2}$ tests which demonstrated a statistically significant correlation between the two groups (Table III; $\chi^{2}=60.071 ; \mathrm{P}<0.001$ ).

\section{Discussion}

The ADAM29 gene is a novel biomarker for esophageal cancer, and is a member of the ADAMs family of single-pass transmembrane and secreted metalloendopeptidases. Different members of the ADAMs family have been detected from cells, transgenic animals and other experimental systems in previous studies (14). Currently, $>40$ species of ADAMs family have been discovered. Their functional regions include the polypeptide domain, metalloproteinase domain, protein-disintegrating domain and cysteine-rich domain, whereby each domain has its associated functions (33). The ADAMs family plays an important role in the occurrence, invasion and metastasis of malignancies, also known as metalloproteinase depolymerization $(21,34)$. They participate in cell signal transduction, adhesion and degradation of extracellular matrix (involving membrane fusion, shedding 
Table III. Association between ADAM29 and FAM135B proteins.

FAM135B

\begin{tabular}{lccc}
\cline { 2 - 4 } ADAM29 & Positive & Negative \\
\hline Positive & 70.0 & & 7.0 \\
Negative & 9.0 & & 34.0 \\
$\chi^{2}$ & & 60.071 & \\
P-value & & $<0.001$ & \\
\hline
\end{tabular}

ADAM29, A Disintegrin and Metalloprotease Domain 29; FAM135B, Family with sequence similarity 135 -member B.

of cytokines and growth factors), the control of cell migration and regulation of other biological processes (21). ADAM29 has the ability to change the surroundings of processes such as cell proliferation, angiogenesis, apoptosis and invasion, which are strongly influenced by the surrounding microenvironment of the tumor, thus contributing to tumor growth and dissemination (35). Furthermore, Noël et al (35) demonstrated that the ADAM29 gene is involved in the regulation of cell proliferation, angiogenesis, apoptosis, cell invasion and other microenvironments. The ADAM29 gene has been reported to be highly expressed in esophageal cancer compared with the corresponding normal tissue (14), and overexpressed in breast cancer (22), lung cancer (23), gastric cancer (24), colorectal cancer (25) and melanoma (26). Mutations of the ADAM29 gene predominantly occur in somatic cells, in the prolysin and propeptide domains, and are associated with increased collagen adhesion of types I and IV compared with wild-type ADAM29 (26). Somatic aberrations are predominantly involved in the Wnt, cell cycle and Notch signaling pathways (14). Brim et al demonstrated that ADAM29 increased the migration ability of cancer cells and caused chromosomes aberrations, resulting in colorectal tumor (25). ADAM29 was also demonstrated to be differently expressed, predominantly in the cytoplasmic binding domain (15).

Through analysis of the staining degree and range of ADAM29 in normal esophageal tissues and esophageal lesions, the present study demonstrated that the expression level of ADAM29 is positively associated with the severity of esophageal epithelial cell lesions. This suggests that ADAM29 may be involved in the evolution process of malignant transformation of esophageal lesions, and a high increment state of ADAM29 exists in each phase. The present study further investigated the association between ADAM29 protein and the clinical characteristics of patients with esophageal cancer. The expression level of ADAM29 protein of ESCC patients with lymph node metastasis was demonstrated to be higher than that of ESCC patients without lymph node metastasis. The ADAM29 gene is considered to be associated with tumor metastasis via its regulatory role in the malignant behavior of ESCC. Furthermore, the results of the present study demonstrated that ADAM29 protein is increasingly expressed in late clinical stage and high pathological grade, in patients with ESCC. The positive expression levels of ADAM29 protein may be associated with malignant transformation of ESCC, which can predict the likelihood of transformation from precancerous lesions to esophageal carcinoma. The results of the present study further indicate that ADAM29 protein expression is closely associated with the occurrence and development of esophageal lesions, which corresponds to the results of previous studies.

The ADAMs family plays an important role in the ErbB (epidermal growth factor) signaling pathway by hydrolyzing a variety of Erb ligand types. The ADAMs family can activate the Erb ligand and cause the shedding of extracellular functional regions $(36,37)$. This particular role has become an important target for the development of new drugs in the Erb signaling pathway. Therefore, the targeted therapy strategy for ADAMs will become an important breakthrough in anti-ErbB signaling. Currently, there are no ADAMs inhibitor used in clinical practice, however; studies on inhibiting ADAM-mediated reactions have entered clinical trials. For example, ADAM10 and ADAM17 participate in the Notch signaling pathway $(21,37)$. Maloum et al (38) demonstrated that ADAM29 gene expression could be detected in order to predict the effect of oral administration of fludarabine and cyclophosphamide on patients with chronic lymphocytic leukemia, and that ADAM29 could guide individualized treatment.

The FAM135B gene is located in chromosome 8 and has been reported to be differentially expressed in healthy people with tuberculosis (27), mental illness (39) and autism (40). To the best of our knowledge, Song et al (14) first reported that the FAM135B gene is a tumor-associated gene for esophageal cancer, by whole-genome sequencing and other technologies. Subsequently, a number of studies have indicated that increased FAM135B expression can enhance the malignant phenotype of ESCC, reflected by the promotion of cell proliferation, metastasis and invasion. Furthermore, high FAM135B expression may be associated with endogenous phosphoglyceraldehyde dehydrogenase $(28,41)$. This suggests that FAM135B is closely associated with the occurrence and development of ESCC. Dong et al (42) demonstrated the function and mechanism behind FAM135B in esophageal cancer, FAM135B promotes inflammation by inducing granulin (GRN; a protein-coding gene located on chromosome 17, q21.32.) precursor secretion and activating the PI3K/AKT/mTOR signaling pathway; moreover, FAM135B and GRN form positive feedback regulation to enhance their respective cancer-promoting functions. The present study demonstrated that there was positive expression of FAM135B in various processes of esophageal precancerous lesions, and occasional or low expression in normal esophageal tissue. Furthermore, the expression of FAM135B was positively associated with the degree of malignant lesions. The results of the present study suggest that FAM135B may be involved in the evolution of malignant transformation of esophageal lesions. Furthermore, the present study assessed the clinical characteristics of patients with esophageal cancer and demonstrated that the expression of FAM135B was positively associated with clinical stage and pathological grade. This experiment demonstrated that high expression of FAM135B could enhance the malignant degree of esophageal lesions. The present study demonstrated that the positive expression of FAM135B in ESCC 

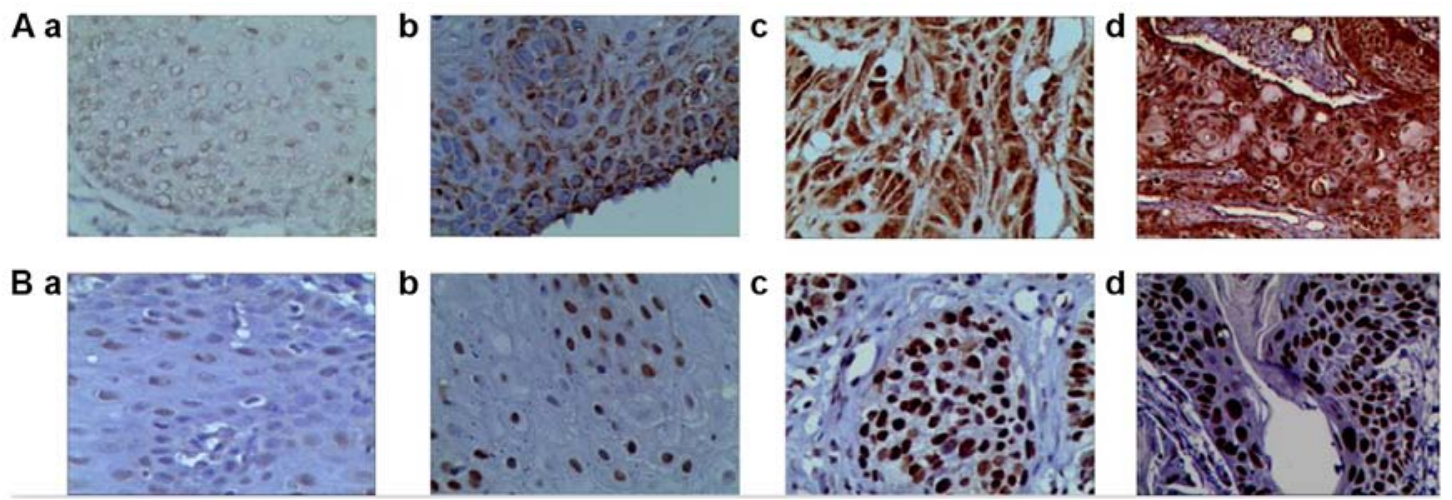

Figure 1. ADAM29 and FAM135B protein expression using H\&E staining, under microscope (magnification, x400). (A) ADAM29 protein; and (B) FAM135B protein. (a) ESCC (brown); (b) esophageal high-grade intraepithelial neoplasia (yellow-brown); (c) esophageal low-grade intraepithelial neoplasia (yellow or light yellow); and (d) normal esophageal mucosal epithelium (light yellow or no coloring). ADAM29, a disintegrin and metalloprotease domain 29; FAM135B, family with sequence similarity 135-member B; H\&E, hematoxylin and eosin; ESCC, esophageal squamous cell carcinoma.
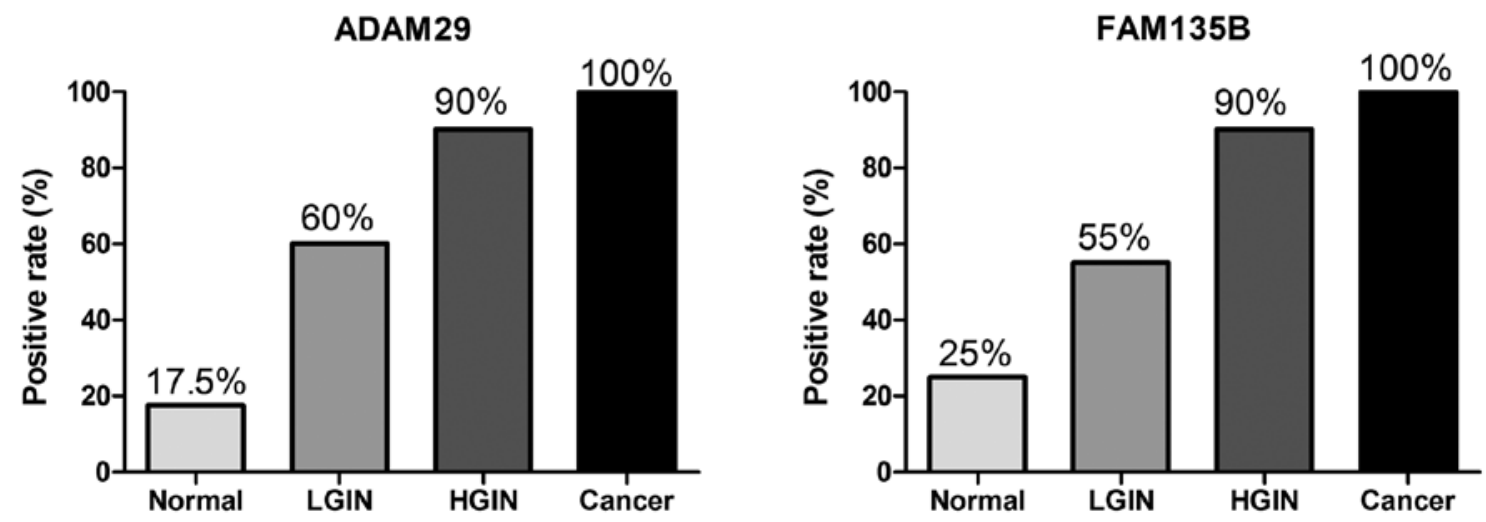

Figure 2. Positive rates of ADAM29 and FAM135B expression in various pathological grades of esophageal cancer. ADAM29, A Disintegrin And Metalloprotease Domain 29; FAM135B, Family with sequence similarity 135-member B; Normal, normal squamous epithelium; LGIN, low-grade intraepithelial neoplasia; HGIN, high-grade intraepithelial neoplasia; Cancer, esophageal squamous cell carcinoma. The expression levels of ADAM29 and FAM135B proteins were associated with the pathological grade.

patients with lymph node metastasis was higher than that of patients without lymph node metastasis. This suggests that the FAM135B gene may be involved in the invasion and migration of different types of esophageal tumor, and in the evolution of normal esophageal tissues into ESCC, which is closely associated with the occurrence, development and invasiveness of esophageal cancer. FAM135B may be useful for the diagnosis of early stage esophageal cancer and precancerous lesions. The present study assessed the association between the FAM135B gene and esophageal cancer, however, further studies are required in order to better understand the underlying molecular mechanisms involved.

Overall, ADAM29 and FAM135B were significantly differentially expressed in different esophageal tissues. Furthermore, the results of the present study demonstrated that they are closely associated with the occurrence and development of ESCC. In addition, a significant correlation was demonstrated between ADAM29 and FAM135B. Both ADAM29 and FAM135B are expected to be the important biological markers for the diagnosis of early stage ESCC and precancerous lesions, and for further contribution to guiding early treatment.

The present study poses a number of limitations due to the selection of biopsy specimens from some out-patient patients with esophageal intraepithelial neoplasia, resulting in incomplete clinical data and a small tissue sample size. First, the present study failed to perform multiple experimental methods in order to verify the results obtained. Secondly, the present study failed to assess the association of ADAM29 and FAM13B expressions with smoking or alcohol consumption. However, Song et al (14) demonstrated that ESCC development is associated with alcohol consumption, through genomic analyses. Thirdly, some specimens were obtained by biopsy in the present study; however, post-surgical specimens are considered to be more credible. It is important to collect more complete experimental images for further studies, in order to increase the reliability of the experimental results. The present study investigated the association between the clinical characteristics of patients with esophageal cancer and the expression of ADAM29 and FAM13B proteins in esophageal cancer tissue samples; however, further analyses on the association between protein expression and the clinic-pathological features in normal tumor-adjacent tissues is required in future studies. The present study is only a retrospective study in some parts of China and the sample size is relatively small, thus future research requires analyses in different populations and larger samples. 


\section{Acknowledgements}

Not applicable.

\section{Funding}

The present study was funded by the National ' 863 ' Project Foundation of China (grant no. 2014AA020901).

\section{Availability of data and materials}

The datasets used and/or analyzed during the current study are available from the corresponding author on reasonable request.

\section{Authors' contributions}

TW performed the experiments, analyzed the data and drafted the initial manuscript. XL, SH and SJ collected specimens and performed the experiments. YW designed the study and performed the quality control. All authors participated in the review of the final manuscript.

\section{Ethics approval and consent to participate}

The present study was approved by the People's Liberation Army 82nd Hospital Ethics Committee. All patients participating in the study (or their family members) have signed informed consent prior to the implementation of the study.

\section{Patient consent for publication}

Not applicable.

\section{Competing interests}

The authors declare that they have no competing interests.

\section{References}

1. Bray F, Ferlay J, Soerjomataram I, Siegel RL, Torre LA and Jemal A: Global cancer statistics 2018 GLOBOCAN estimates of incidence and mortality worldwide for 36 cancers in 185 countries. CA Cancer J Clin 68: 394-424, 2018.

2. Wei KR, Lian QH, Liu J and Liang ZH: Prevalence of esophageal cancer. Chin J Int Med 51: 156-158, 2012 (In Chinese).

3. Malhotra GK, Yanala U, Ravipati A, Follet M, Vijayakumar M and Are C: Global trends in esophageal cancer. J Surg Oncol 115 564-579, 2017.

4. Torre LA, Bray F, Siegel RL, Ferlay J, Lortet-Tieulent J and Jemal A: Global cancer statistics, 2012. CA Cancer J Clin 65 : 87-108, 2015.

5. Zhang Y: Epidemiology of esophageal cancer. World J Gastroenterol 19: 5598-5606, 2013.

6. Zhou MG, Wang XF, Hu JP, Li GL, Chen WQ, Zhang SW, Wan X, Wang LJ, Xiang C, Hu YS and Yang GH: Geographical distribution of cancer mortality in China, 2004-2005. Zhonghua Yu Fang Yi Xue Za Zhi 44: 303-308, 2010 (In Chinese).

7. Li QW, Yuan GJ, Du YX, Pan CN and He Y: Analysis of the prevalence and treatment of esophageal cancer in huai an area. J Clin Oncol 17: 142-145, 2012.

8. Siegel R, Naishadham D and Jemal A: Cancer statistics, 2012. CA Cancer J Clin 62: 10-29, 2012.

9. Wang GQ, Jiao GG, Chang FB, Fang WH, Song JX, Lu N, Lin DM, Xie YQ and Yang L: Long-term results of operation for 420 patients with early squamous cell esophageal carcinoma discovered by screening. Ann Thorac Surg 77: 1740-1744, 2004.
10. Aminian A, Panahi N, Mirsharifi R, Karimian F, Meysamie A, Khorgami Z and Alibakhshi A: Predictors and outcome of cervical anastomotic leakage after esophageal cancer surgery. J Cancer Res Ther 7: 448-453, 2011.

11. Wang GQ, Zhang YM and He S: Present situation and prospect of diagnosis and treatment of early esophageal cancer and precancerous lesions in China. Chin Cancer 18: 690-694, 2009 (In Chinese).

12. Siewert JR and Ott K: Are squamous and adenocarcinomas of the esophagus the same disease. Semin Radiat Oncol 17: 38-44, 2007.

13. Hao J and Shao K: Epidemiological status, diagnosis and treatment of esophageal cancer in China and China's future countermeasures. Chin J Cancer 501-504, 2011 (In Chinese).

14. Song Y, Li L, Ou Y, Gao Z, Li E, Li X, Zhang W, Wang J, Xu L, Zhou Y, et al: Identification of genomic alterations in oesophageal squamous cell cancer. Nature 509: 91-95, 2014.

15. Cerretti DP, DuBose RF, Black RA and Nelson N: Isolation of two novel metalloproteinase-disintegrin (ADAM) cDNAs that show testis-specific gene expression. Biochem Biophys Res Commun 263: 810-815, 1999.

16. Edwards DR, Handsley MM and Pennington CJ: The ADAM metalloproteinases. Mol Aspects Med 29: 258-289, 2008.

17. Wang F, Xu R,Zhu P, Hu J, Ying B, Zhao S and Li C: Preliminarily functional analysis of a cloned novel human gene ADAM29. Sci China C Life Sci 44: 392-399, 2001.

18. Oria VO, Lopatta P, Schmitz T, Preca BT, Nyström A, Conrad C, Bartsch JW, Kulemann B, Hoeppner J, Maurer J, et al: ADAM9 contributes to vascular invasion in pancreatic ductal adenocarcinoma. Mol Oncol 13: 456-479, 2019.

19. Luo ML, Zhou Z, Sun LC, Sun L, Yu L, Sun L, Liu J, Yang Z, Ran Y, Yao Y and $\mathrm{Hu} \mathrm{H}$ : An ADAM12 and FAK positive feedback loop amplifies the interaction signal of tumor cells with extracellular matrix to promote esophageal cancer metastasis. Cancer Lett 422: 118-128, 2018.

20. Li W, Wang D, Sun X, Zhang Y, Wang L and Suo J: ADAM17 promotes lymph node metastasis in gastric cancer via activation of the Notch and Wnt signaling pathways. Int J Mol Med 43: 914-926, 2019.

21. Mochizuki S and Okada Y: ADAMs in cancer cell proliferation and progression. Cancer Sci 98: 621-628, 2007.

22. Zhang B, Li Y, Li L, Chen M, Zhang C, Zuo XB, Zhou FS, Liang B, Zhu J, Li P, et al: Association study of susceptibility loci with specific breast cancer subtypes in Chinese women. Breast Cancer Res Treat 146: 503-514, 2014.

23. Shan Q, Lou X, Xiao T, Zhang J, Sun H, Gao Y, Cheng S, Wu L, $\mathrm{Xu} \mathrm{N}$ and Liu S: A cancer/testis antigen microarray to screen autoantibody biomarkers of non-small cell lung cancer. Cancer Lett 328: 160-167, 2013.

24. Costa NR, Paulo P, Caffrey T, Hollingsworth MA and Santos-Silva F: Impact of MUC1 mucin down regulation in the phenotypic characteristics of MKN45 gastric carcinoma cell line. PLoS One 6: e26970, 2011.

25. Brim H, Abu-Asab MS, Nouraie M, Salazar J, Deleo J, Razjouyan H, Mokarram P, Schaffer AA, Naghibhossaini F and Ashktorab H: An integrative CGH, MSI and candidate genes methylation analysis of colorectal tumors. PLoS One 9: e82185, 2014.

26. Wei X, Moncada-Pazos A, Cal S, Soria-Valles C, Gartner J, Rudloff U, Lin JC; NISC Comparative Sequencing Program, Rosenberg SA, López-Otín C and Samuels Y: Analysis of the disintegrin-metalloproteinases family reveals ADAM29 and ADAM7 are often mutated in melanoma. Hum Mutat 32: E2148-E2175, 2011.

27. Oki NO, Motsinger-Reif AA, Antas PR, Levy S, Holland SM and Sterling TR: Novel human genetic variants associated with extrapulmonary tuberculosis A pilot genome wide association study. BMC Res Notes 4: 28, 2011.

28. Zhou C, Ye M, Ni S, Li Q, Ye D, Li J, Shen Z and Deng H: DNA methylation biomarkers for head and neck squamous cell carcinoma. Epigenetics 13: 398-409, 2018.

29. Rice TW: Esophageal cancer staging. Korean J Thorac Cardiovasc Surg 48: 157-163, 2015.

30. Rice TW, Rusch VW, Ishwaran $\mathrm{H}$ and Blackstone EH; Worldwide Esophageal Cancer Collaboration: Cancer of the esophagus and esophagogastric junction Data-driven staging for the seventh edition of the American joint committee on cancer/international union against cancer staging manuals. Cancer 116: 3763-3773, 2010. 
31. Edge SB, Byrd DR, Compton CC, et al (eds): AJCC cancer staging manual. 7th edition. Springer, New York, NY, 2010.

32. Zhou ZJ, Xie JL, Wei P and Zhou XG: Pathologic subtyping of primary lymphoma of breast and prognostic analysis. Zhonghua Bing Li Xue Za Zhi 46: 618-622, 2017 (In Chinese; Abstract available in Chinese from the publisher).

33. Seals DF and Courtneidge SA: The ADAMs family of metalloproteases: Multidomain proteins with multiple functions. Genes Dev 17: 7-30, 2003.

34. Rocks N, Paulissen G, El Hour M, Quesada F, Crahay C, Gueders M, Foidart JM, Noel A and Cataldo D: Emerging roles of ADAM and ADAMTS metalloproteinases in cancer. Biochimie 90: 369-379, 2008.

35. Noël A, Gutiérrez-Fernández A, Sounni NE, Behrendt N, Maquoi E, Lund IK, Cal S, Hoyer-Hansen G and López-Otín C: New and paradoxical roles of matrix metalloproteinases in the tumor microenvironment. Front Pharmacol 3: 140, 2012.

36. Lu X, Lu D, Scully M and Kakkar V: ADAM proteins-therapeutic potential in cancer. Curr Cancer Drug Targets 8: 720-732, 2008.

37. Murphy G: The ADAMs Signalling scissors in the tumour microenvironment. Nat Rev Cancer 8: 929-941, 2008.

38. Maloum K, Settegrana C, Chapiro E, Cazin B, Leprêtre S, Delmer A, Leporrier M, Dreyfus B, Tournilhac O, Mahe B, et al: IGHV gene mutational status and LPL/ADAM29 gene expression as clinical outcome predictors in CLL patients in remission following treatment with oral fludarabine plus cyclophosphamide. Ann Hematol 88: 1215-1221, 2009.
39. Quilter CR, Sargent CA, Bauer J, Bagga MR, Reiter CP, Hutchinson EL, Southwood OI, Evans G, Mileham A, Griffin DK and Affara NA: An association and haplotype analysis of porcine maternal infanticide A model for human puerperal psychosis. Am J Med Genet B Neuropsychiatr Genet 159: 908-927, 2012.

40. Tsang KM, Croen LA, Torres AR, Kharrazi M, Delorenze GN, Windham GC, Yoshida CK, Zerbo $\mathrm{O}$ and Weiss LA: A genome-wide survey of transgenerational genetic effects in autism. PLoS One 8: e76978, 2013

41. Lin JQ, Huang ZJ, Chen ZR, et al: Relationship between FAM135B gene and lymph node metastasis of esophageal squamous cell carcinoma. Chin J Exp Surg 6: 1170-1171, 2018 (In Chinese).

42. Dong DZ: The Function and Mechanism of FAM135B in the Deveopment of esophageal squamous cell carcinoma (unpublished PhD thesis). Peking Union Medical College, 2018.

(i) (9) This work is licensed under a Creative Commons Attribution-NonCommercial-NoDerivatives 4.0 International (CC BY-NC-ND 4.0) License. 